\title{
Insights into multidrug-resistant $K$. pneumoniae urinary tract infections: From susceptibility to mortality
}

\author{
IONELA-LARISA MIFTODE $^{1 *}$, EDUARD VASILE NASTASE $^{1 *}$, RADU-ȘTEFAN MIFTODE $^{2}$, \\ EGIDIA GABRIELA MIFTODE ${ }^{1}$, LUMINIȚA SMARANDA IANCU ${ }^{3}$, CĂTĂLINA LUNCĂ $\breve{C}^{3}$, \\ DANA-TEODORA ANTON PĂDURARU ${ }^{4}$, IRINA-IULIANA COSTACHE ${ }^{2}$, \\ CELINA-SILVIA STAFIE ${ }^{3^{*}}$ and OLIVIA-SIMONA DORNEANU ${ }^{3}$ \\ Departments of ${ }^{1}$ Infectious Diseases (Internal Medicine II), ${ }^{2}$ Cardiology (Internal Medicine I), \\ ${ }^{3}$ Preventive Medicine and Interdisciplinarity, and ${ }^{4}$ Mother and Child Medicine, Faculty of Medicine, \\ 'Gr. T. Popa' University of Medicine and Pharmacy, 700115 Iasi, Romania
}

Received May 31, 2021; Accepted June 30, 2021

DOI: $10.3892 /$ etm.2021.10520

\begin{abstract}
The incidence of urinary tract infections (UTIs) caused by Klebsiella pneumoniae has exhibited an increasing trend and has become a high burden for many public health systems, especially in hospital settings. Multidrug resistance associated with the production of extended-spectrum $\beta$-lactamases (ESBL) among $K$. pneumoniae isolates is endemic in Southeastern Europe. We retrospectively analyzed 75 cases admitted to 'St. Parascheva' Clinical Hospital of Infectious Diseases in Iasi, Romania, during the first 6 months of 2019 (January 1 to June 30), who had a confirmed diagnosis of $K$. pneumoniae UTI at discharge. From a total of 75 patients, $34(45.3 \%)$ presented $\mathrm{ESBL}^{+}$K. pneumoniae. The mean age was 66 years (70.1 for the $\mathrm{ESBL}^{+}$patients vs. 62.6 for the $\mathrm{ESBL}^{-}$patients, $\left.\mathrm{P}=0.0365\right)$. There was a symmetrical sex distribution ( 37 men vs. 38 women). Of these, 22 men had $\mathrm{ESBL}^{+} K$. pneumoniae UTIs, compared to only 15 with an $\mathrm{ESBL}^{-}$strain, $\mathrm{P}=0.0087$. Another risk factor for $\mathrm{ESBL}^{+}$ $K$. pneumoniae UTIs was the presence of hospitalization in the past 6 months; $20(58.82 \%)$ patients with $\mathrm{ESBL}^{+}$infections were previously hospitalized, compared to only 5 (12.19\%)
\end{abstract}

Correspondence to: Dr Radu-Ștefan Miftode, Department of Cardiology (Internal Medicine I), Faculty of Medicine, 'Gr. T. Popa' University of Medicine and Pharmacy, 16 Universitatii Street, 700115 Iasi, Romania

E-mail: radu.miftode@yahoo.com

Professor Egidia Gabriela Miftode, Department of Infectious Diseases (Internal Medicine II), Faculty of Medicine, 'Gr. T. Popa' University of Medicine and Pharmacy, 16 Universitatii Street, 700115 Iasi, Romania

E-mail: emiftode@yahoo.co.uk

*Contributed equally

Key words: ESBL-producing, K. pneumoniae, susceptibility, risk factors, antibiotics patients with $\mathrm{ESBL}^{-}$strains, $\mathrm{P}<0.0001$. The urinary catheter carriers presented an increased prevalence of $\mathrm{ESBL}^{+}$ infections (15/34 vs. 5/41, $\mathrm{P}=0.0012)$. Regarding mortality, $\mathrm{ESBL}^{+}$infections caused 6 fatalities, compared to only 1 death in the $\mathrm{ESBL}^{-}$group, $\mathrm{P}=0.0166 . \mathrm{ESBL}^{+} K$. pneumoniae strains represent an important cause of healthcare-related UTIs, with a significantly higher mortality rate compared to ESBL $^{-}$strains. Early identification and adequate management of the risk factors incriminated in $\mathrm{ESBL}^{+} \mathrm{UTIs}$ should be a priority for physicians in order to limit the dissemination of the ESBL-producing strains and thus to improve the outcome of these patients.

\section{Introduction}

Klebsiella pneumoniae (KP) is a relatively common component of the normal microbiota, having the capacity to colonize various tissues and anatomical structures (e.g., distal urethra, upper respiratory tract, or the gastrointestinal tract). It is considered an opportunistic pathogen, being incriminated in the etiology of severe infections in hospitalized patients, especially in those immunocompromised or who present severe comorbidities $(1,2)$.

The multidrug-resistance of KP, in particular to carbapenems due to the production of carbapenemases, has increased dramatically over the past 10 years and has become an important worldwide burden for the public health systems $(3,4)$. First recognized almost four decades ago, extended-spectrum $\beta$-lactamases (ESBL) are enzymes produced by multiple gram-negative bacteria, being significantly involved in the resistance of these bacteria to almost all $\beta$-lactam antibiotics, except for cephamycins and carbapenems $(5,6)$.

The ESBL mechanism of action is based upon their capacity to hydrolyze the $\beta$-lactam ring of the third-generation cephalosporins and aztreonam, but are inhibited by clavulanic acid (7).

The ESBL producers can also develop co-resistance to other classes of antibiotics, such as fluoroquinolones, cotrimoxazole or aminoglycosides, which are frequently used in the treatment of urinary tract infections (UTIs), thus inducing a severe limitation to their therapeutic approach $(8,9)$. This 
aspect is due to the coexistence, on large plasmids, of the genes that encode ESBL with similar genes for resistance to other antimicrobial agents, subsequently creating a multidrug-resistant phenotype that is more and more associated with ESBL-producing Enterobacteriaceae (10). As a result, the judicious choice of empirical antibiotic therapy for UTI treatment is of paramount importance as it must be approached in accordance to the risk factors for infections due to ESBL-producing K. pneumoniae (ESBL $\left.{ }^{+} \mathrm{KP}\right)$, such as recurrent UTIs, diabetes mellitus (DM), previous antibiotic usage, female sex or urinary catheterization (11).

The epidemiological magnitude determined by ESBL-producing $\mathrm{KP}\left(\mathrm{ESBL}^{+} \mathrm{KP}\right)$ isolates is based on their significantly increased morbidity and mortality rates, prolonged hospitalization, and important financial burden (12). Additionally, there is a major concern regarding the increasing prevalence of $\mathrm{ESBL}^{+} \mathrm{KP}^{\mathrm{s}}$; a multicenter study conducted over a period of 10 years revealed a worldwide prevalence of $16 \%$, with higher rates among patients hospitalized in intensive care units (ICU) (13). A similar increasing trend over time was noted in the case of colonization with $\mathrm{ESBL}^{+} \mathrm{KP}$, with rates in ICU patients ranging from $2.6 \%$ in the US to almost $50 \%$ in India (14). However, is it likely that the real prevalence is higher than the reported numbers, due to the paucity of reported data and difficulties in laboratory assessment as many testing kits fail to detect all ESBL producers.

In this challenging context, healthcare providers aim to find the ideal, thin balance between an effective antibiotic treatment against $\mathrm{ESBL}^{+} \mathrm{KP}$ and to avoid the selection of new multidrug-resistant strains, the current paradigm being focused on preventing their spread both in the community and in healthcare facilities.

\section{Patients and methods}

Study design and population. We conducted this retrospective study aiming to assess the incidence, risk factors and antibiotic susceptibility of $K$. pneumoniae urinary strains isolated from patients admitted to the 'St. Parascheva' Clinical Hospital of Infectious diseases in Iasi, Romania, a University Clinic with 300 beds, between January 1, 2019 and June 30, 2019.

We performed a comparative assessment between patients with $\mathrm{ESBL}^{+} \mathrm{KP}$ infections and the ESBL-negative (ESBL) ones, by dividing them into two distinct cohorts: group 1, patients with $\mathrm{ESBL}^{+} \mathrm{KP}$ UTIs and group 2, patients with non-ESBL producing KP (ESBL- KP) UTIs. The main objectives were to assess the individual epidemiological characteristics, risk factors for antibiotic resistance and overall patient mortality.

In the present study, we included all hospitalized patients presenting with UTIs, with the following additional inclusion criteria: i) suggestive clinical syndrome (dysuria, pollakiuria); ii) pyuria $\left[\geq 10\right.$ white blood cell count $\left.(\mathrm{WBC}) / \mathrm{mm}^{3}\right]$; iii) isolation of $K$. pneumoniae in urine culture $\left[\geq 10^{5}\right.$ colony forming units $(\mathrm{CFU}) / \mathrm{ml})]$. We excluded patients with $K$. pneumoniae colonization and those with a urinary CFU count $<10^{5} / \mathrm{ml}$.

Study and laboratory measurements. The following antimicrobial agents were tested by disk diffusion method: Ampicillin + sulbactam, cefixime, cefuroxime, cefotaxime, cefepime, ceftazidime, cefuroxime, cefoxitin, gentamicin, amikacin, ciprofloxacin, imipenem, meropenem, ertapenem, and colistin. EUCAST clinical breakpoint table v8.0 (15) was used for the interpretation of the minimal inhibitory concentrations and zone diameters.

We analyzed several parameters from the patient medical hospital records. Demographic characteristics (including age, sex), a full medical history, previous conditions (hospitalizations in the past 6 months, history of antibiotic use within the previous 30 days), clinical aspects (underlying diseases, invasive procedures) were fully recorded. Important comorbidities (e.g., chronic renal failure, cardiovascular diseases, diabetes mellitus, malignancy, chronic liver diseases, neurological pathologies) were previously documented or diagnosed during the current hospitalization. Charlson comorbidity index was used for assessing the overall mortality risk, based on patients coexisting comorbidities. Recurrent UTI in adults is defined as 2 or more UTIs in the last 6 months or 3 or more UTIs in the last 12 months. Diabetes mellitus was defined as a fasting blood glucose level $\geq 126 \mathrm{mg} / \mathrm{dl}$, a HbA1c $\geq 6.5 \%$ or current consumption of antidiabetic medication. Obesity was established in all patients presenting a body mass index $\geq 30 \mathrm{~kg} / \mathrm{m}^{2}$.

Routine investigations as part of the admission approach (complete blood count, inflammatory markers), assessment of the antibiotic susceptibility, hospitalization length, therapeutic management (including inappropriate empirical antibiotics and targeted single therapy/association of antibiotics) and clinical outcome were registered as well.

Statistical analysis. Categorical variables are presented as numbers and percentages, with continuous variables being presented as means and standard deviations. We used the $95 \%$ confidence interval in parameter estimation. Independent t-tests were used to compare continuous variables, while Chi-squared tests were used to compare categorical variables. Mann-Whitney U test was used for abnormally distributed categorical variables. A P-value of $<0.05$ was considered statistically significant for all of the analyses. Statistical analysis was performed with SPSS v20 (IBM Corp.) and EpiInfo v7.2 softwares (developer: Centers for Disease Control and Prevention).

\section{Results}

A total of 75 patients met the inclusion criteria for the study. Group 1 was comprised of 34 patients (45.3\%) who had an $\mathrm{ESBL}^{+} \mathrm{KP} \mathrm{UTI}$, while group 2 was made up of 41 patients $(54.7 \%)$ with an $\mathrm{ESBL}^{-} \mathrm{KP}$ UTI. Out of the $34 \mathrm{ESBL}^{+} \mathrm{KP}$ cases, $6(17.6 \%)$ were OXA48-producers.

Mean age of the total participants was $66 \pm 17.72$ years (70.1 \pm 18.68 for group 1 vs. $62.6 \pm 7.21$ for group $2 ; \mathrm{P}=0.0365)$. The mean age of patients infected with OXA $48^{+}$strains was 69.3 years. We found a symmetrical sex distribution in the entire cohort ( 37 males and 38 females), but with a significant difference concerning the sex distribution in the two groups: (64.7\% males in group 1 vs. $36.5 \%$ in group $2, \mathrm{P}=0.087$ ) (Table I). Out of the 6 OXA48-producing cases, 4 were males.

The baseline patient characteristics are summarized in Table II. In addition to the demographic aspects, we also found statistically significant differences between the two groups concerning the underlying conditions, such as the recurrent 
Table I. Age distribution of the patients with K. pneumoniae UTI.

\begin{tabular}{lcccr}
\hline Age interval & $\mathrm{ESBL}^{+} \mathrm{KP}(\mathrm{n}=34)$ & $\mathrm{ESBL}^{-} \mathrm{KP}(\mathrm{n}=41)$ & P-value & OR (95\% CI) \\
\hline 0-20 years & $1(2.9 \%)$ & $5(12.1 \%)$ & 0.0855 & $0.218(0.024-1.966)$ \\
$21-50$ years & $3(8.8 \%)$ & $4(9.7 \%)$ & 0.4528 & $0.895(1.186-4.307)$ \\
$51-70$ years & $10(29.4 \%)$ & $17(41.4 \%)$ & 0.1464 & $0.588(0.224-1.543)$ \\
$\geq 71$ years & $20(58.8 \%)$ & $15(36.5 \%)$ & $\mathbf{0 . 0 3 0 3}$ & $2.476(0.974-6.294)$ \\
Mean age & 70.1 & 62.6 & $\mathbf{0 . 0 3 6 5}$ &
\end{tabular}

All data are expressed as n (\%). UTI, urinary tract infections; KB, Klebsiella pneumoniae; ESBL, extended-spectrum $\beta$-lactamase; ESBL ${ }^{+}$ KP, ESBL-producing K. pneumoniae; ESBL- KP, non-ESBL producing KP. OR, odds ratio; CI, confidence interval. Significant P-values are presented in bold print.

Table II. Baseline characteristics of the patients with K. pneumoniae UTI.

\begin{tabular}{|c|c|c|c|c|}
\hline Characteristics & $\mathrm{ESBL}^{+} \mathrm{KP}(\mathrm{n}=34)$ & $\operatorname{ESBL}^{-} \mathrm{KP}(\mathrm{n}=41)$ & P-value & OR $(95 \% \mathrm{CI})$ \\
\hline \multicolumn{5}{|l|}{ Demographics } \\
\hline Age, median (IQR) & 70.1 & 62.6 & 0.0365 & \\
\hline Male sex & $22(64.7 \%)$ & $15(36.5 \%)$ & 0.0080 & $3.177(1.231-8.200)$ \\
\hline \multicolumn{5}{|l|}{ Previous conditions } \\
\hline Hospitalizations in the past 6 months & $20(58.8 \%)$ & $5(12.1 \%)$ & $<0.0001$ & $10.285(3.23-32.752)$ \\
\hline Antibiotic treatment in the last 30 days & $9(26.4 \%)$ & $4(9.7 \%)$ & 0.0340 & $3.330(0.923-12006)$ \\
\hline \multicolumn{5}{|l|}{ Underlying conditions } \\
\hline Chronic kidney disease & $8(23.5 \%)$ & $3(7.31 \%)$ & 0.0296 & $3.897(0.944-16.085)$ \\
\hline Recurrent UTIs & $12(35.2 \%)$ & $7(17.0 \%)$ & 0.0404 & $2.694(0.903-7.765)$ \\
\hline Cardiovascular diseases & $24(70.5 \%)$ & $20(48.7 \%)$ & 0.0309 & $2.520(0.966-6.573)$ \\
\hline Diabetes mellitus & $10(29.4 \%)$ & $13(31.7 \%)$ & 0.4180 & $0.897(0.334-2.411)$ \\
\hline Chronic liver disease & $6(17.6 \%)$ & $13(31.7 \%)$ & 0.0880 & $0.461(0.153-1.386)$ \\
\hline Malignancy & $11(32.3 \%)$ & $5(12.1 \%)$ & 0.0203 & $3.443(1.058-11.201)$ \\
\hline Cerebrovascular disease & $11(32.3 \%)$ & $9(21.9 \%)$ & 0.1630 & $1.700(0.606-4.768)$ \\
\hline Obesity & $7(20.5 \%)$ & $9(21.9 \%)$ & 0.4470 & $0.921(0.303-2.804)$ \\
\hline Charlson comorbidity index, median (IQR) & 5.7 & 3.5 & 0.0005 & \\
\hline Urinary catheterization & $15(44.1 \%)$ & $5(12.1 \%)$ & 0.0011 & $5.684(1.791-18.036)$ \\
\hline \multicolumn{5}{|l|}{ Laboratory findings } \\
\hline Hemoglobin $<11.7 \mathrm{~g} / \mathrm{dl}$ & $16(47.0 \%)$ & $11(26.8 \%)$ & 0.0385 & $2.424(0.923-6.361)$ \\
\hline White blood cells $>10,000 / \mathrm{mm}^{3}$ & $19(55.8 \%)$ & $19(46.3 \%)$ & 0.2113 & $1.466(0.588-3.657)$ \\
\hline Erythrocyte sedimentation rate $>12 \mathrm{~mm} / \mathrm{h}$ & $24(32.0 \%)$ & $36(48.0 \%)$ & $\mathbf{0 . 0 3 7 2}$ & $0.333(0.101-1.097)$ \\
\hline \multicolumn{5}{|l|}{ Treatment } \\
\hline Inappropriate empirical therapy & $18(52.9 \%)$ & $19(46.3 \%)$ & 0.3334 & $1.226(0.496-3.025)$ \\
\hline Targeted therapy & $17(47.0 \%)$ & $22(53.6 \%)$ & & \\
\hline
\end{tabular}

All data are expressed as n (\%). UTI, urinary tract infections; KB, Klebsiella pneumoniae; ESBL, extended-spectrum $\beta$-lactamase; ESBL ${ }^{+}$ KP, ESBL-producing K. pneumoniae; ESBL- KP, non-ESBL producing KP. IQR, interquartile range; OR, odds ratio; CI, confidence interval. Significant $\mathrm{P}$-values are presented in bold print.

UTIs, chronic kidney disease, cardiovascular pathologies and malignancies, which were more commonly associated with $\mathrm{ESBL}^{+} \mathrm{KP}$. This aspect was furtherly confirmed by an increased Charlson comorbidity index among patients with $\mathrm{ESBL}^{+}$KP UTI (5.7 vs. 3.5, $\left.\mathrm{P}=0.0005\right)$. Moreover, both the presence of an indwelling urinary catheter ( 15 vs. $5, P=0.0011)$ and previous hospitalizations in the past six months (20 vs. 5 ,
$\mathrm{P}<0.0001)$ or a history of antibiotic use within the last 30 days ( 9 vs. $4, \mathrm{P}=0.034$ ) were significant predictors for the presence of $\mathrm{ESBL}^{+} \mathrm{KP}$ (Table II).

Another important aspect of the study was to highlight the relationship between the incidence of $\mathrm{ESBL}^{+} \mathrm{KP}$ and the use of specific antibiotics. Noteworthy, we observed that patients without ESBL-producing strains were more commonly treated 
Table III. Antibiotic treatment of the enrolled patients with K. pneumoniae UTI.

\begin{tabular}{lcccc}
\hline Variables & ESBL $^{+} \mathrm{KP}(\mathrm{n}=34)(\%)$ & $\mathrm{ESBL}^{-} \mathrm{KP}(\mathrm{n}=41)(\%)$ & P-value & OR $(95 \% \mathrm{CI})$ \\
\hline Single antibiotic therapy & $7(20.5)$ & $16(39.0)$ & $\mathbf{0 . 0 4 6 2}$ & $0.405(0.143-1.147)$ \\
2 antibiotic therapy & $16(47.0)$ & $18(43.9)$ & 0.3949 & $1.135(0.455-2.830)$ \\
$\geq 3$ antibiotic therapy & $11(32.3)$ & $7(17.0)$ & 0.0683 & $2.323(0.784-6.877)$ \\
Most commonly used antibiotics & & & & \\
Ciprofloxacin & $6(17.6)$ & $21(51.2)$ & $\mathbf{0 . 0 0 1 4}$ & $0.204(0.069-0.597)$ \\
Ampicillin + sulbactam & $6(17.6)$ & $13(31.7)$ & 0.0882 & $0.461(0.153-1.386)$ \\
3rd generation cephalosporins & $6(17.6)$ & $21(51.2)$ & $\mathbf{0 . 0 0 1 4}$ & $0.204(0.069-0.597)$ \\
Carbapenems & $18(52.9)$ & $6(14.6)$ & $\mathbf{0 . 0 0 0 2}$ & $6.562(2.190-19.657)$ \\
Amikacin & $19(55.8)$ & $7(17.0)$ & $\mathbf{0 . 0 0 0 2}$ & $6.152(2.135-17.728)$ \\
Trimethoprim/sulfamethoxazole & $5(14.7)$ & $8(19.5)$ & 0.302 & $0.711(0.209-2.417)$ \\
Colistin & $6(17.6)$ & $1(2.4)$ & $\mathbf{0 . 0 1 6 6}$ & $8.571(0.977-75.178)$ \\
\hline
\end{tabular}

UTI, urinary tract infections; KB, Klebsiella pneumoniae; ESBL, extended-spectrum $\beta$-lactamases; ESBL ${ }^{+} \mathrm{KP}_{\text {, ESBL-producing }}$ K. pneumoniae; ESBL- KP, non-ESBL producing KP. OR, odds ratio; CI, confidence interval. Significant P-values are presented in bold print.

Table IV. Evolution of the patients with K. pneumoniae UTI.

\begin{tabular}{lcccc}
\hline Variables & $\mathrm{ESBL}^{+} \mathrm{KP}(\mathrm{n}=34)$ & $\mathrm{ESBL}^{-} \mathrm{KP}(\mathrm{n}=41)$ & P-value & OR $(\mathrm{CI})$ \\
\hline Length of hospital stay (IQR) & 13.1 days & 9.4 days & $\mathbf{0 . 0 0 1 2}$ & \\
Mortality & $6(17.6 \%)$ & $1(2.4 \%)$ & $\mathbf{0 . 0 1 6 6}$ & $8.571(0.977-75.178)$ \\
\hline
\end{tabular}

UTI, urinary tract infections; KB, Klebsiella pneumoniae; ESBL, extended-spectrum $\beta$-lactamases; $\mathrm{ESBL}^{+} \mathrm{KP}$, ESBL-producing K. pneumoniae; ESBL- KP, non-ESBL producing KP; IQR, interquartile range; OR, odds ratio; CI, confidence interval. Significant $\mathrm{P}$-values are presented in bold print.

with one single antibiotic $(\mathrm{P}=0.046)$, the use of regimens consisting of multiple antibiotic molecules ( $\geq 3$ antibiotics) being observed in the lot infected with $\mathrm{ESBL}^{+} \mathrm{KP}(32.3$ vs. $17 \%, \mathrm{P}=0.0683$ ). Keeping in mind the resistance profile of $\mathrm{ESBL}^{+} \mathrm{KP}$, we identified a positive correlation between the administration of ciprofloxacin, amikacin, colistin or 3rd generation cephalosporins and the absence of ESBL producers. Contrary, the presence of $\mathrm{ESBL}^{+} \mathrm{KP}$ was significantly associated with the large-scale use of carbapenems, indirectly reflecting both the susceptibility profile and the severity of infection (Table III).

In terms of evolution, the patients with $\mathrm{ESBL}^{+} \mathrm{KP}$ presented a severe prognosis, characterized both by prolonged hospitalization (13.1 vs. 9.4 days, $\mathrm{P}=0.0012)$ and by a much higher mortality rate compared to those without ESBL-producing strains (17.6 vs. $2.4 \%, \mathrm{P}=0.0166$; Table IV).

The differences between ESBL and non-ESBL susceptibility profiles are illustrated in Fig. 1, revealing the quasi-total resistance of $\mathrm{ESBL}^{+} \mathrm{KP}$ to cephalosporins, but also to certain other classes of antibiotics, such as fluoroquinolones (ciprofloxacin) or aminoglycosides (gentamicin).

Furthermore, our study aimed to identify the risk factors for a poor outcome among the included patients. In this regard, we performed a comparative analysis between the surviving patients and the ones who were deceased. When assessing certain data from the personal history, we observed that elderly patients $>70$ years present a high risk for a fatal outcome $(\mathrm{P}=0.019)$, a similar negative evolution being also associated with previous antibiotic therapy $(\mathrm{P}=0.008)$ or other hospitalizations in the past six months $(\mathrm{P}=0.021)$. Very importantly, even if the share of severe comorbidities (except for diabetes mellitus) was higher among the deceased patients, the differences did not reach the threshold of statistical significance.

Evaluating the classic risk factors for UTIs, we found a significant association between the mortality rate and the presence of an indwelling urinary catheter $(\mathrm{P}=0.044)$ or a prolonged hospitalization $(\mathrm{P}=0.033)$. Moreover, a severe course of the infection, translated as the initiation of an aggressive antibiotic treatment (e.g., carbapenems) was significantly correlated with an increased fatality rate $(\mathrm{P}=0.018)$. Finally, when analyzing the ESBL production among deceased patients, we noted that 6 out of 7 fatalities presented an $\mathrm{ESBL}^{+} \mathrm{KP}$ infection $(\mathrm{P}=0.016)$, suggesting the important negative prognostic value of these strains in the outcome of patients with UTI (Table V).

\section{Discussion}

The current situation of antimicrobial resistance has reached a crucial point since 2005 . In UTIs, multidrug-resistant Enterobacteriaceae, including ESBL-producing microorganisms, can be readily encountered, emerging as a growing 
Table V. Risk factors for in-hospital mortality of the patients with K. pneumoniae UTI.

\begin{tabular}{|c|c|c|c|c|}
\hline Factors & $\begin{array}{c}\text { Deceased } \\
\mathrm{N}=7\end{array}$ & $\begin{array}{l}\text { Surviving } \\
\mathrm{N}=68\end{array}$ & P-value & OR $(95 \% \mathrm{CI})$ \\
\hline \multicolumn{5}{|l|}{ Demographics } \\
\hline Age $>70$ years & $6(85.7 \%)$ & $29(42.6 \%)$ & 0.019 & $8.069(0.95-75.734)$ \\
\hline Age, median (IQR) & 75.8 & 65 & 0.142 & \\
\hline Male sex & $3(42.8 \%)$ & $34(50.0 \%)$ & 0.370 & $0.750(0.155-3.607)$ \\
\hline \multicolumn{5}{|l|}{ Previous conditions } \\
\hline Hospitalizations in the past 6 months & $5(71.4 \%)$ & $20(29.4 \%)$ & 0.021 & $6.000(1.073-33.534)$ \\
\hline Previous antibiotic therapy & $4(57.1 \%)$ & $9(13.2 \%)$ & 0.008 & $8.740(1.673-45.656)$ \\
\hline \multicolumn{5}{|l|}{ Comorbidities } \\
\hline Chronic kidney disease & $2(28.5 \%)$ & $9(13.2 \%)$ & 0.165 & $2.622(0.44-15.604)$ \\
\hline Cardiovascular pathology & $5(71.4 \%)$ & $39(57.3 \%)$ & 0.256 & $1.859(0.336-10.266)$ \\
\hline Diabetes mellitus & $2(28.5 \%)$ & $22(32.3 \%)$ & 0.838 & $0.836(0.15-4.655)$ \\
\hline Chronic liver disease & $2(28.5 \%)$ & $17(25.0 \%)$ & 0.836 & $1.200(0.212-6.763)$ \\
\hline Malignancy & $2(28.5 \%)$ & $15(22.0 \%)$ & 0.695 & $1.413(0.248-8.029$ \\
\hline Cerebrovascular disease & $2(28.5 \%)$ & $18(26.4 \%)$ & 0.904 & $1.111(0.197-6.242)$ \\
\hline Obesity & $2(28.5 \%)$ & $14(20.5 \%)$ & 0.623 & $1.542(0.27-8.808)$ \\
\hline Charlson comorbidity index, median (IQR) & 6 & 4.3 & 0.153 & \\
\hline Urinary catheterization & $4(57.1 \%)$ & $16(23.5 \%)$ & 0.044 & $4.333(0.876-21.429)$ \\
\hline Length of hospital stay (IQR) & 15.2 & 10.6 & $\mathbf{0 . 0 3 3}$ & \\
\hline Carbapenem treatment after laboratory diagnosis & $5(71.4 \%)$ & $19(27.9 \%)$ & 0.018 & $6.447(1.15-36.124)$ \\
\hline \multicolumn{5}{|l|}{ ESBL production } \\
\hline $\mathrm{ESBL}^{+}$ & $6(17.6 \%)$ & $28(82.3 \%)$ & 0.0166 & $8.571(0.977-75.178)$ \\
\hline $\mathrm{ESBL}^{-}$ & $1(2.4 \%)$ & $40(97.5 \%)$ & & \\
\hline
\end{tabular}

UTI, urinary tract infections; KB, Klebsiella pneumoniae; ESBL, extended-spectrum $\beta$-lactamases; ESBL ${ }^{+}$KP,ESBL-producing K.pneumoniae; ESBL- KP, non-ESBL producing KP; IQR, interquartile range; OR, odds ratio; CI, confidence interval. Significant P-values are presented in bold print.

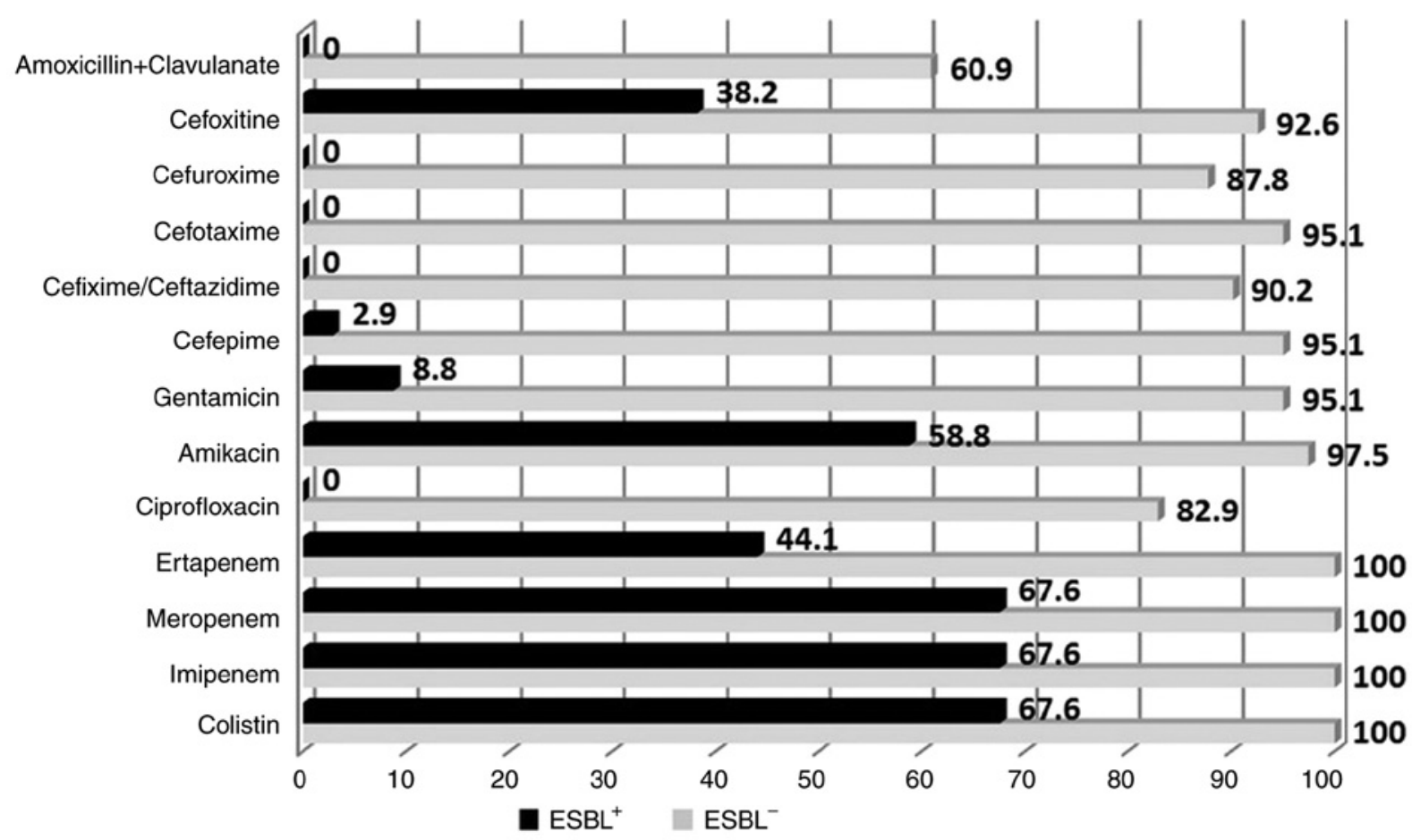

Figure 1. $\mathrm{ESBL}^{+}$and ESBL- KP susceptibility profiles (\%) to various classes of antibiotics. KB, Klebsiella pneumoniae; ESBL, extended-spectrum $\beta$-lactamase; $\mathrm{ESBL}^{+} \mathrm{KP}$, ESBL-producing $K$. pneumoniae; ESBL- KP, non-ESBL producing KP. 
challenge in many infectious disease clinical setups (16). In addition to the fact that patients with ESBL-producing bacteria are generally prone to a more severe course of infection compared to those without ESBL isolates, another serious issue is represented by the paucity of antibiotics that can be used for the treatment of multidrug-resistant $K$. pneumoniae (KP) UTIs. The persistent and often inappropriate exposure of KP strains to various $\beta$-lactam antibiotics has created the background for dynamic mutations and enhanced synthesis of $\beta$-lactamases in these bacteria, thus inducing multidrug resistance even against the extremely useful, broad-spectrum, 3rd generation cephalosporins $(17,18)$.

Previous studieshave reported a variable, geographic-related incidence of $\mathrm{ESBL}^{+} \mathrm{KP}$. This regional variability is explained by several factors, ranging from the specific, local pattern of resistance to some socio-economic aspects, such as healthcare access or household income, with higher rates being prevalent in developing, low-income countries from Asia or Eastern Europe (19-21). Given the scarce data in the literature concerning ESBL-producing strains in the North East region of Romania (22), our study aimed to unravel the local resistance profile in an area with certain socio-epidemiological particularities $(23,24)$.

We found a very high prevalence of ESBL-producing isolates (45.3\%) among all K. pneumoniae strains detected in urinary specimens from a university hospital serving a region with $\sim 4$ million inhabitants.

Urinary catheterization is a well-known risk factor for UTIs, this aspect being confirmed by multiple previous studies, starting from Platt et al study in 1986, when about $20 \%$ of UTIs were associated with Foley catheterization, due to contamination at the insertion of the catheter (25). Additionally, Lee et al (26) found that ESBL-producing bacteria were approximately 2.4 times more frequent in patients with Foley catheterization. In line with these findings, our study revealed that 20 patients $(26.6 \%)$ had an indwelling urinary catheter, which was significantly associated with the presence of $\mathrm{ESBL}^{+}$ $\mathrm{KP}\left(15 \mathrm{ESBL}^{+} \mathrm{KP}\right.$ vs. $\left.5 \mathrm{ESBL}^{-} \mathrm{KP}, \mathrm{P}=0.0011\right)$.

A past history of exposure to antibiotics and hospitalizations in the last 6 months may represent another risk factor for the emergence of ESBL-producing strains. In the literature, particularly the use of cefaclor and cefminox was incriminated for the emergence of resistant strains (26). In our study, we identified a previous antibiotic exposure in 26.4\% of the patients with $\mathrm{ESBL}^{+} \mathrm{KP}$, compared to only $9.7 \%$ with an ESBL- KP $(\mathrm{P}=0.0340)$. Regarding recent contact with healthcare facilities, we also found a noteworthy difference among the two groups, 20 (58.8\%) for the ESBL producers vs. $5(12.1 \%)$ for the non-ESBL producers $(\mathrm{P}<0.0001)$ presenting a documented hospitalization in the last six months. Similarly, Yilmaz et al (27) found that $90 \%$ of the patients with an ESBL producer had a history of previous hospitalization in the past three months vs. only $16.7 \%$ for the ESBL-negative ones.

Basically, when reviewing the literature (28-30), we noted a plethora of risk factors for infection with ESBL-producing bacteria that were also identified in our research, such as recent antibiotic use, residence in long-term care facilities, recent hospitalization, old age and male sex. In our study, elderly patients ( $>70$ years), male sex, hospitalization in the past 6 months, previous antibiotic treatment, chronic renal failure, recurrent UTIs, urinary catheterization, longer hospital stay, underlying cardiovascular pathologies, malignancies, and anemia were considerably more prevalent among the patients with $\mathrm{ESBL}^{+}$KP UTIs. The latter aspects may be explained by the mutual and dynamic relationship between serious comorbidities and the occurrence and severity of KP infections. Frail patients with impaired immunity due to a severe associated pathology are more prone to develop a KP infection, and vice versa, the KP infection may negatively influence the outcome of such a patient. For example, a recent study highlighted the significant incidence and poor prognosis of carbapenemase-producing KP infections among patients with hematological malignancies or aplastic anemia (31).

Antimicrobial susceptibility was investigated for all isolates. Our results demonstrate that, among the $\mathrm{ESBL}^{+} \mathrm{KP}$ patients, susceptibility rates to ciprofloxacin, gentamicin, amikacin, imipenem, meropenem and colistin were extremely low: $0,8.9,58.9,67.7,67.7$ and $67.7 \%$, respectively. On the other hand, the ESBL- KP strains showed lower susceptibility to amoxicillin + clavulanic acid (61\%), ciprofloxacin $(83 \%)$ and cefuroxime $(87.8 \%)$, while high susceptibility was identified for carbapenems (100\%), amikacin (97.6\%), gentamicin (95.1\%), cefepime (95.1\%), ceftazidime (90.3\%), cefotaxime (95.1\%), cefixime (90.3\%) and cefoxitin (92.7\%), these findings being in accordance with other results that have identified a similar resistance pattern, thus raising awareness both on the adequate therapeutic approach and nosocomial or community spread of $\mathrm{ESBL}^{+} \mathrm{KP}$ (32). It is noteworthy to point out that the antibiotic combination between a $\beta$-lactam and an aminoglycoside is synergistic and is widely used as a first-line therapy prior to antibiogram results, especially in patients with established risk factors for multidrug-resistant infections. Given the local susceptibility profile, in addition to a $\beta$-lactam, in our setting we prefer the use of amikacin instead of gentamicin given the higher resistance rate associated with the latter.

However, despite the similarities, there are also some susceptibility-related differences, mainly due to particular local factors. Even if some of the results from a Pakistani study (33) were similar to ours concerning the susceptibility profile of ciprofloxacin, amikacin, imipenem and meropenem they also found a significantly lower susceptibility to gentamicin for the ESBL- KP (63.1 vs. $95.1 \%$ in our study). A more recent multicentric study from the same country furtherly confirmed this regional pattern of resistance, thus bringing attention to the geographical polymorphism of $\mathrm{ESBL}^{+} \mathrm{KP}$ (34). Coinciding results were found in another study performed by Müller-Schulte et al (35) regarding the differences between the susceptibility profiles depending on the ESBL production in a sub-Saharan country; for the $\mathrm{ESBL}^{+} \mathrm{KP}$ the susceptibility to ciprofloxacin, gentamicin, meropenem, cefuroxime, ceftazidime and cefotaxime was 38,21,100, 0, 0 and $0 \%$, respectively, while for the ESBL- KP the susceptibility was 88, 94, 100, 100, 100 and $100 \%$, respectively.

Numerous studies have examined whether ESBL production has an adverse effect on clinical outcomes. Although most of the reported data revealed an association between unfavorable outcome and infections with $\mathrm{ESBL}^{+} \mathrm{KP}(36-40)$, other studies have failed to demonstrate such a link (41). In 
our patients we identified a significant association between the presence of $\mathrm{ESBL}^{+} \mathrm{KP}$ and the morality rate $(\mathrm{P}=0.016)$. Noteworthy, of the six patients with OXA48-producing $K$. pneumoniae UTI, we recorded two deaths.

Furthermore, we found that the risk factors for all-cause mortality were similar to those for $\mathrm{ESBL}^{+} \mathrm{KP}$ infection and included: Age $>70$ years $(\mathrm{P}=0.019)$, hospitalizations in the past 6 months $(\mathrm{P}=0.024)$, previous antibiotic treatment $(\mathrm{P}=0.003)$, urinary catheterization $(\mathrm{P}=0.044)$, length of hospital stay $(\mathrm{P}=0.033)$, carbapenem treatment based on antibiogram result $(\mathrm{P}=0.018)$ and $\mathrm{ESBL}$ production $(\mathrm{P}=0.0166)$.

In terms of therapeutic approach, we could not quantify accurately the superiority of any particular antibiotic regimen in the prognosis of $K$. pneumoniae UTI mainly due to the heterogenicity of the regimens (often consisting of at least 3 different antibiotics), but also due to some patient-related specific aspects which led to the use of only certain antibiotics. As a general algorithm, before initiating antibiotic treatment in $\mathrm{ESBL}^{+}$patients, of paramount importance, is to assess the in vitro susceptibilities, the degree of the infection's source control and, finally, the clinical condition of each patient (7).

Carbapenems exhibit the broadest spectrum of $\beta$-lactam antibiotics, presenting the highest potency against Gram-negative bacteria, but being also characterized by stability to hydrolysis by the majority of $\beta$-lactamases. The majority of available data have demonstrated that carbapenem treatment is associated with improved outcomes in patients with severe $\mathrm{ESBL}^{+} \mathrm{KP}$ infections and remains the 'gold standard' especially in critically ill patients $(7,42,43)$. The research spectrum of $\mathrm{ESBL}^{+} \mathrm{KP}$ has focused mainly on meropenem or imipenem, although a recently published multicentric study also assessed the efficacy of ertapenem (44), finding similar cure rates $(90.6 \%$ with ertapenem and $75.5 \%$ with other carbapenems in empiric and 89.8 and $82.6 \%$ in targeted treatment), without significant differences concerning mortality rates. Importantly, in our study we observed a rather low $\mathrm{ESBL}^{+} \mathrm{KP}$ susceptibility rate to ertapenem (44.1\%), compared to imipenem or meropenem (67.6\% each), thus outlining bleak future prospects when compared to a $100 \%$ susceptibility rate to both ertapenem and imipenem reported less than two decades ago $(45,46)$.

Our study has limitations related to the relatively small size of the research group and the retrospective nature. Given the continuously increasing trend of $\mathrm{ESBL}^{+} \mathrm{KP}$ prevalence, this means that our results could underestimate the actual burden of these strains, therefore requiring careful interpretation of the data. Despite these limitations, our study provides several insights on the association between the $\mathrm{ESBL}^{+} \mathrm{KP}$ and the outcome of the UTI patients with this etiology, as well as the implications regarding susceptibility profiles and local risk factors from the Northeast region of Romania. In conclusion, our data may have a significant impact for further antibiotic prescription in patients with communityand/or hospital-acquired UTIs, mainly focusing on the early identification or even prevention of certain risk factors associated with ESBL-producing KP.

\section{Acknowledgements}

Not applicable.

\section{Funding}

No funding was received.

\section{Availability of data and materials}

Data used in this original study were obtained from the patient personal medical records admitted to the 'St. Parascheva' Clinical Hospital of Infectious Diseases in Iasi, Romania. Any further information regarding the present study is available from the corresponding authors upon reasonable request.

\section{Authors' contributions}

All authors presented an equal contribution to this paper. ILM, OSD and EGM designed the study and collected data from the included patients. OSD, CL and LSI performed the susceptibility tests and other laboratory determinations. DTAP, EVN, IIC and CSS analyzed data and performed the statistics. ILM, OSD and RSM analyzed and wrote the Results and Discussion sections including the literature review. RSM and EVN prepared the manuscript, translated it and managed all the correspondence for publishing. All authors were actively involved in conceiving the paper and they read and approved the final version of the manuscript for publication.

\section{Ethics approval and patient consent}

This study was approved by the local Ethics Commission of 'St. Parascheva' Clinical Hospital of Infectious Diseases in Iasi, Romania (approval no. 8/2019) and it was conducted according to the ethical guidelines of the 1975 Declaration of Helsinki Principles. Patient consent was not required due to the retrospective character of the study.

\section{Patient consent for publication}

Not applicable.

\section{Competing interests}

There are no competing interests regarding the authors of this research.

\section{References}

1. Shimasaki T, Seekatz A, Bassis C, Rhee Y, Yelin RD, Fogg L, Dangana T, Cisneros EC, Weinstein RA, Okamoto K, et al: Increased relative abundance of Klebsiella pneumoniae carbapenemase-producing Klebsiella pneumoniae within the gut microbiota is associated with risk of bloodstream infection in long-term acute care hospital patients. Clin Infect Dis 68: 2053-2059, 2019.

2. Navon-Venezia S, Kondratyeva $\mathrm{K}$ and Carattoli $\mathrm{A}$. Klebsiella pneumoniae: A major worldwide source and shuttle for antibiotic resistance. FEMS Microbiol Rev 41: 252-275, 2017.

3. Lee BY, Bartsch SM, Wong KF, McKinnell JA, Slayton RB, Miller LG, Cao C, Kim DS, Kallen AJ, Jernigan JA and Huang SS: The potential trajectory of carbapenem-resistant Enterobacteriaceae, an emerging threat to health-care facilities, and the impact of the centers for disease control and prevention toolkit. Am J Epidemiol 183: 471-479, 2016. 
4. Braun SD, Dorneanu OS, Vremeră T, Reißig A, Monecke S and Ehricht R: Carbapenemase-producing Enterobacteriaceae A 2-year surveillance in a hospital in Iaşi, Romania. Future Microbiol 11: 391-401, 2016

5. Bradford PA: Extended-spectrum beta-lactamases in the 21st century: Characterization, epidemiology, and detection of this important resistance threat. Clin Microbiol Rev 14: 933-951, 2001.

6. European Committee on Antimicrobial Susceptibility Testing (EUCAST): EUCAST guidelines for detection of resistance mechanisms and specific resistances of clinical and/or epidemiological importance. Version 2.01.EUCAST, Sweden, 2017. https://www.eucast.org/fileadmin/src/media/PDFs/ EUCAST_files/Resistance_mechanisms/EUCAST_detection_of_ resistance_mechanisms_170711.pdf. Accessed Febrary 20, 2021.

7. Pana ZD and Zaoutis T: Treatment of extended-spectrum $\beta$-lactamase-producing Enterobacteriaceae (ESBLs) infections: What have we learned until now? F1000Res 7: F1000 Faculty Rev-1347, 2018.

8. Paterson DL and Bonomo RA: Extended-spectrum beta-lactamases: A clinical update. Clin Microbiol Rev 18: 657-686, 2005.

9. Miftode E, Dorneanu O, Leca D, Teodor A, Mihalache D, Filip $\mathrm{O}$ and Luca V: Antimicrobial resistance profile of $E$. coli and Klebsiella spp. From urine in the infectious diseases hospital Iaşi. Rev Med Chir Soc Med Nat Iasi 112: 478-482, 2008 (In Romanian)

10. Brolund A, Edquist PJ, Mäkitalo B, Olsson-Liljequist B, Söderblom T, Wisell KT and Giske CG: Epidemiology of extended-spectrum $\beta$-lactamase-producing Escherichia coli in Sweden 2007-2011. Clin Microbiol Infect 20: O344-O352, 2014.

11. Rodríguez-Baño J, Alcalá JC, Cisneros JM, Grill F, Oliver A, Horcajada JP, Tórtola T, Mirelis B, Navarro G, Cuenca M, et al: Community infections caused by extended-spectrum betalactamase-producing Escherichia coli. Arch Intern Med 168: 1897-1902, 2008.

12. Maslikowska JA, Walker SA, Elligsen M, Mittmann N, Palmay L, Daneman N and Simor A: Impact of infection with extended-spectrum $\beta$-lactamase-producing Escherichia coli or Klebsiella species on outcome and hospitalization costs. J Hosp Infect 92: 33-41, 2016

13. Morrissey I, Hackel M, Badal R, Bouchillon S, Hawser S and Biedenbach D: A review of ten years of the study for monitoring antimicrobial resistance trends (SMART) from 2002 to 2011. Pharmaceuticals (Basel) 6: 1335-1346, 2013.

14. Biehl LM, Schmidt-Hieber M, Liss B, Cornely OA and Vehreschild MJ: Colonization and infection with extended spectrum beta-lactamase producing Enterobacteriaceae in high-risk patients-review of the literature from a clinical perspective. Crit Rev Microbiol 42: 1-16, 2016.

15. EUCAST Clinical breakpoints-breakpoints and guidance, 2018. https://www.eucast.org/clinical_breakpoints/. Accessed December 10, 2019.

16. Kader AA and Kumar A: Prevalence and antimicrobial susceptibility of extended-spectrum beta-lactamase-producing Escherichia coli and Klebsiella pneumoniae in a general hospital. Ann Saudi Med 25: 239-242, 2005.

17. Sarojamma V andRamakrishna V:Prevalence of ESBL-producing Klebsiella pneumoniae isolates in tertiary care hospital. ISRN Microbiol 2011: 318348, 2011.

18. Pitout JD and Laupland KB: Extended-spectrum beta-lactamase-producing Enterobacteriaceae: An emerging public-health concern. Lancet Infect Dis 8: 159-166, 2008.

19. Goossens H; MYSTIC Study Group (Europe): MYSTIC program: Summary of European data from 1997 to 2000. Diagn Microbiol Infect Dis 41: 183-189, 2001.

20. Hoşoğlu S, Gündes S, Kolayli F, Karadenizli A, Demirdağ K, Günaydin M, Altindis M, Caylan $\mathrm{R}$ and Ucmak $\mathrm{H}$ : Extended-spectrum beta-lactamases in ceftazidime-resistant Escherichia coli and Klebsiella pneumoniae isolates in Turkish hospitals. Indian J Med Microbiol 25: 346-350, 2007.

21. Xiong Z, Zhu D, Zhang Y and Wang F: Extended-spectrum beta-lactamase in Klebsiella pneumoniae and Escherichia coli isolates. Zhonghua Yi Xue Za Zhi 82: 1476-1479, 2002 (In Chinese)

22. Miftode E, Dorneanu O, Badescu A, Ghibu L, Leca D, Vremera T and Mereuţă A: Emergence of a new group CTX-M enzyme in Romania and risk factors for extended spectrum beta-lactamase producing E. coli infections. Rev Med Chir Soc Med Nat Iasi 116 : $477-480,2012$
23. Anton-Paduraru DT, Miftode EG, Iliescu ML, Pricop C, Carauleanu A and Boiculese LV: Knowledge of adolescent girls regarding sexually transmitted diseases a study in a rural area from North-Eastern Romania. Rev Cercet Interv Soc 69: 143-155, 2020.

24. Costache II, Miftode E, Mitu O and Aursulesei V: Sex differences in cardiovascular risk factors in a rural community from North Romania Region. Rev Cercet Interv Soc 55: 204-214, 2016.

25. Platt R, Polk BF, Murdock B and Rosner B: Risk factors for nosocomial urinary tract infection. Am J Epidemiol 124: 977-985, 1986.

26. Lee DS, Lee CB and Lee SJ: Prevalence and risk factors for extended spectrum beta-lactamase-producing uropathogens in patients with urinary tract infection. Korean J Urol 51: 492-497, 2010.

27. Yilmaz E, Akalin H, Ozbey S, Kordan Y, Sinirtaş M, Gürcüoglu E, Ozakin C, Heper Y, Mistik R, and Helvaci S: Risk factors in community-acquired/onset urinary tract infections due to extended-spectrum beta-lactamase-producing Escherichia coli and Klebsiella pneumoniae. J Chemother 20: 581-585, 2008.

28. Vading M, Nauclér P, Kalin M and Giske CG: Invasive infection caused by Klebsiella pneumoniae is a disease affecting patients with high comorbidity and associated with high long-term mortality. PLoS One 13: e0195258, 2018.

29. Nguyen ML, Toye B, Kanji S and Zvonar R: Risk factors for and outcomes of bacteremia caused by extended-spectrum $\beta$-lactamase-producing Escherichia coli and Klebsiella species at a Canadian tertiary care hospital. Can J Hosp Pharm 68: 136-143, 2015.

30. Koksal E, Tulek N, Sonmezer MC, Temocin F, Bulut C, Hatipoglu C, Erdinc FS and Ertem G: Investigation of risk factors for community-acquired urinary tract infections caused by extended-spectrum beta-lactamase Escherichia coli and Klebsiel species. Investig Clin Urol 60: 46-53, 2019.

31. Tofas P, Skiada A, Angelopoulou M, Sipsas N, Pavlopoulou I, Tsaousi S, Pagoni M, Kotsopoulou M, Perlorentzou S, Antoniadou A, et al: Carbapenemase-producing Klebsiella pneumoniae bloodstream infections in neutropenic patients with haematological malignancies or aplastic anaemia: Analysis of 50 cases. Int J Antimicrob Agents 47: 335-339, 2016.

32. Parveen RM, Khan MA, Menezes GA, Harish BN, Parija SC and Hays JP: Extended-spectrum $\beta$-lactamase producing Klebsiella pneumoniae from blood cultures in Puducherry, India. Indian J Med Res 134: 392-395, 2011

33. Ullah F, Malik SA and Ahmed J: Antimicrobial susceptibility pattern and ESBL prevalence in Klebsiella pneumoniae from urinary tract infections in the North-West of Pakistan. Afr J Microbiol Res 3: 676-680, 2009.

34. Abdullah FE, Mushtaq A, Irshad M, Rauf H, Afzal N and Rasheed A: Current efficacy of antibiotics against Klebsiella isolates from urine samples-a multi-centric experience in Karachi. Pak J Pharm Sci 26: 11-15, 2013.

35. Müller-Schulte E, Tuo MN, Akoua-Koffi C, Schaumburg F and Becker SL: High prevalence of ESBL-producing Klebsiella pneumoniae in clinical samples from central Côte d'Ivoire. Int J Infect Dis 91: 207-209, 2020.

36. Lautenbach E, Patel JB, Bilker WB, Edelstein PH and Fishman NO: Extended-spectrum beta-lactamase-producing Escherichia coli and Klebsiella pneumoniae: Risk factors for infection and impact of resistance on on outcomes. Clin Infect Dis 32: 1162-1171, 2001.

37. Shanthi M and Sekar U: Extended spectrum beta lactamase producing Escherichia coli and Klebsiella pneumoniae: Risk factors for infection and impact of resistance on outcomes. J Assoc Physicians India 58 (Suppl): S41-S44, 2010.

38. MacVane SH, Tuttle LO and Nicolau DP: Impact of extended-spectrum $\beta$-lactamase-producing organisms on clinical and economic outcomes in patients with urinary tract infection. J Hosp Med 9: 232-238, 2014.

39. Shamsrizi P, Gladstone BP, Carrara E, Luise D, Cona A, Bovo C and Tacconelli E: Variation of effect estimates in the analysis of mortality and length of hospital stay in patients with infections caused by bacteria-producing extended-spectrum beta-lactamases: A systematic review and meta-analysis. BMJ Open 10: e030266, 2020.

40. Vata A, Hunea IM, Dorneanu O, Harja-Alexa IA, Plesca C, Leonte-Enache G, Ciocan A, Ghiciuc CM, Esanu I, Manolache M and Luca CM: Biochemical changes and risk factors in the prognosis of antibiotics susceptibility in urinary tract infections. Rev Chim 70: 1822-1825, 2019. 
41. Kola A, Maciejewski O, Sohr D, Ziesing S and Gastmeier P Clinical impact of infections caused by ESBL-producing E. coli and $K$. pneumoniae. Scand J Infect Dis 39: 975-982, 2007.

42. Bassetti M, Peghin M and Pecori D: The management of multidrug-resistant Enterobacteriaceae. Curr Opin Infect Dis 29: 583-594, 2016.

43. Tamma PD, Han JH, Rock C, Harris AD, Lautenbach E, Hsu AJ, Avdic E and Cosgrove SE; Antibacterial Resistance Leadership Group: Carbapenem therapy is associated with improved survival compared with piperacillin-tazobactam for patients with extended-spectrum $\beta$-lactamase bacteremia. Clin Infect Dis 60: 1319-1325, 2015.

44. Gutiérrez-Gutiérrez B, Bonomo RA, Carmeli Y, Paterson DL, Almirante B, Martínez-Martínez L, Oliver A, Calbo E, Peña C, Akova M, et al: Ertapenem for the treatment of bloodstream infections due to ESBL-producing Enterobacteriaceae: A multinational pre-registered cohort study. J Antimicrob Chemother 71: 1672-1680, 2016.
45. Betriu C, Salso S, Sánchez A, Culebras E, Gómez M, Rodriguez-Avial I and Picazo JJ: Comparative in vitro activity and the inoculum effect of ertapenem against Enterobacteriaceae resistant to extended-spectrum cephalosporins. Int J Antimicrob Agents 28: 1-5, 2006.

46. Mody RM, Erwin DP, Summers AM, Carrero HA, Selby EB, Ewell AJ and Moran KA: Ertapenem susceptibility of extended spectrum beta-lactamase-producing organisms. Ann Clin Microbiol Antimicrob 6: 6, 2007.

(i)(9) This work is licensed under a Creative Common Attribution-NonCommercial-NoDerivatives 4.0 International (CC BY-NC-ND 4.0) License. 